\title{
Interactive comment on "JULES-BE:
}

\section{representation of bioenergy crops and harvesting in the Joint UK Land Environment Simulator vn5.1" by Emma W. Littleton et al.}

\section{Emma W. Littleton et al.}

e.w.littleton@exeter.ac.uk

Received and published: 21 November 2019

Thanks to Referee \#1 for their constructive and thoughtful critique which has helped to improve the clarity of the manuscript. Our response to their comments is attached as a PDF.

Please also note the supplement to this comment: 
2019.

GMDD

Interactive

comment 